\title{
Télescope
}

Revue d'analyse comparée en administration publique

\section{Favoriser l'innovation dans l'élaboration des politiques : un nouveau rôle pour les politiciens}

\section{Eva Sørensen}

Volume 19, numéro 2, printemps 2013

L'innovation dans le secteur public : au-delà des discours

URI : https://id.erudit.org/iderudit/1023838ar

DOI : https://doi.org/10.7202/1023838ar

Aller au sommaire du numéro

Éditeur(s)

L'Observatoire de l'administration publique

ISSN

1929-3348 (numérique)

Découvrir la revue

Citer cet article

Sørensen, E. (2013). Favoriser l'innovation dans l'élaboration des politiques : un nouveau rôle pour les politiciens. Télescope, 19(2), 22-37.

https://doi.org/10.7202/1023838ar

\section{Résumé de l'article}

Dans de nombreux pays occidentaux, l'innovation dans le secteur public grimpe au sommet des préoccupations de la recherche et des réformes. L'innovation est de plus en plus vue comme l'une des principales manières d'améliorer l'efficacité et le rendement de la prestation des services publics, mais la nécessité de l'innovation sur le plan des politiques a été largement ignorée. Elle représente pourtant un aspect crucial de l'innovation dans le secteur public. De nouvelles avenues au sein de la théorie de l'innovation et de la théorie de la gouvernance soulignent le rôle important de la collaboration dans la promotion de l'innovation, tant dans le secteur public que privé. Cependant, les efforts consentis afin d'augmenter la capacité du secteur public à mettre en oeuvre des projets de collaboration innovante en matière de politiques nécessitent le développement d'un nouveau rôle pour les politiciens, comme l'illustrent deux études de cas danois portant sur de tels projets et présentées dans cet article. 


\title{
FAVORISER L'INNOVATION DANS L'ÉLABORATION DES POLITIQUES : UN NOUVEAU RÔLE POUR LES POLITICIENS
}

\author{
Par Eva Sørensen, Professeure, Roskilde University, Danemark \\ eva@ruc.dk
}

Traduit de l'anglais

RÉSUMÉ Dans de nombreux pays occidentaux, l'innovation dans le secteur public grimpe au sommet des préoccupations de la recherche et des réformes. L'innovation est de plus en plus vue comme l'une des principales manières d'améliorer l'efficacité et le rendement de la prestation des services publics, mais la nécessité de l'innovation sur le plan des politiques a été largement ignorée. Elle représente pourtant un aspect crucial de l'innovation dans le secteur public. De nouvelles avenues au sein de la théorie de l'innovation et de la théorie de la gouvernance soulignent le rôle important de la collaboration dans la promotion de l'innovation, tant dans le secteur public que privé. Cependant, les efforts consentis afin d'augmenter la capacité du secteur public à mettre en œuvre des projets de collaboration innovante en matière de politiques nécessitent le développement d'un nouveau rôle pour les politiciens, comme l'illustrent deux études de cas danois portant sur de tels projets et présentées dans cet article.

ABSTRACT Public innovation is moving to the top of the research and reform agendas in many Western countries. Innovation is increasingly viewed as an important means to improve the efficiency and effectiveness of public service delivery, but the need for policy innovation has been largely overlooked despite the fact that policy innovation is a key aspect of public innovation. New strands of innovation theory and governance theory highlight the important role of collaboration for promoting public as well as private sector innovation. However, efforts to enhance the public sector's capacity for collaborative policy innovation calls for the development of a new role for politicians, as is illustrated by two Danish case studies of collaborative policy innovation projects. 
À l'heure actuelle, et au sein de nombreux pays occidentaux, l'innovation dans le secteur public occupe une place prépondérante dans les programmes de réformes et de recherche. L'image du secteur public en tant que domaine intrinsèquement non novateur (Downs, 1967) perd du terrain, de même que la notion, lancée par le paradigme de la nouvelle gestion publique (Niskanen, 1987; Hood, 1991; Osborne et Gaebler, 1993), selon laquelle l'innovation dans le secteur public ne peut être stimulée qu'en organisant le secteur public de façon à ce qu'il imite le secteur privé. Le paradigme émergent de la nouvelle gouvernance publique (Osborne, 2010) offre une vision différente de l'innovation. Selon ce paradigme, le secteur public jouit déjà d'une bonne capacité à innover et celle-ci pourrait être favorisée par l'application de formes collaboratives de gestion. Ainsi, une stratégie d'innovation dans le secteur public devrait viser l'amélioration de la capacité d'un secteur à engendrer des collaborations et des partenariats entre institutions et intervenants pertinents des secteurs privé et public, plutôt que de rendre celui-ci plus concurrentiel (Borins, 2001; Newman, Raine et Skelcher, 2001; Mulgan et Albury, 2003; Dente, Bobbio et Spada, 2005; Nambisan, 2008; Eggers et Singh, 2009; Bland et autres, 2010; Bommert, 2010; Sørensen et Torfing, 2011).

Bien que cette approche collaborative offre d'importantes nouvelles pistes de compréhension pour l'amélioration de l'innovation dans le secteur public, elle pose deux problèmes. Premièrement, elle a tendance à diminuer l'importance du rôle crucial que peuvent jouer la hiérarchie et la concurrence dans la promotion de l'innovation. La collaboration ne devrait pas être considérée comme une solution de rechange, mais comme une addition irremplaçable à la hiérarchie et à la concurrence (Sørensen, 2012). Deuxièmement, la nouvelle gestion publique met principalement l'accent sur le rôle potentiel de la collaboration en tant que moteur de l'innovation dans les services, mais néglige de prendre en compte l'influence bénéfique que pourrait avoir la collaboration sur des politiques novatrices. La théorie de l'innovation et la théorie de la gestion engendrées par le secteur privé ont créé un fort héritage théorique et généré une source d'inspiration, tout en plaçant les politiques, et l'innovation dans l'élaboration des politiques, aux confins du débat portant sur l'innovation dans le secteur public. Le présent article a pour objectif de ramener les politiques novatrices au cœur des préoccupations de la recherche dans le domaine de l'innovation dans le secteur public. Les politiques novatrices sont la colonne vertébrale de l'innovation dans le secteur public, d'une part parce qu'il s'agit d'un aspect inséparable de l'innovation dans les services et d'autre part parce que tout système politique a pour objectif de renouveler les visions politiques, les buts et les stratégies qui guident la gestion publique. Une approche collaborative de l'innovation en matière de politiques qui reconnaît le rôle de la hiérarchie et de la concurrence en tant que moteurs de la collaboration innovante montre la nécessité de réévaluer le rôle joué par les politiciens dans la gestion des affaires publiques.

Dans cet article, nous expliquons d'abord les raisons qui font des politiques novatrices l'un des éléments clés de l'innovation dans le secteur public et entreprenons ensuite de décrire différents aspects de l'innovation en matière de politiques. Ensuite, nous montrons pourquoi et comment la collaboration, combinée à la hiérarchie et à la concurrence, devrait être considérée comme un puissant moteur de l'innovation dans le secteur public en général et en matière de politiques en particulier. Suit une description des barrières pouvant entraver la bonne marche de la collaboration. Nous poursuivons en énonçant la manière dont l'augmentation de la collaboration innovante en termes de politiques entraîne un nouveau rôle pour les politiciens. Ce nouveau rôle est illustré par les résultats de deux études de cas 
qui s'intéressent à deux projets danois qui avaient pour objectif de favoriser la collaboration innovante en matière de politiques.

\section{LES POLITIQUES NOVATRICES, UN ÉLÉMENT CLÉ DE L'INNOVATION DANS LE SECTEUR PUBLIC}

L'innovation dans le secteur public se distingue de l'innovation dans le secteur privé par le fait qu'elle est mise en œuvre dans un contexte politique. Malgré ce fait, les innovations en matière de politiques n'ont pas été au cœur des récents débats portant sur la manière de favoriser l'innovation dans ce secteur. Ce peu d'intérêt peut s'expliquer par le fait que le paradigme de la nouvelle gestion publique trouvait principalement son inspiration dans les théories et les pratiques développées dans le contexte non politique du secteur privé. En revanche, le paradigme de la nouvelle gouvernance publique tire son origine de l'approche de la gouvernance publique liée à l'administration publique et dominée par une vision de la gouvernance plus gestionnaire que politique (Osborne et Gaebler, 1993; Warren, 2009).

Ces perspectives font fi du fait que la gouvernance dans le secteur public, dont l'innovation dans ce secteur, est conditionnée par des luttes de pouvoir institutionnalisées entre partis politiques et groupes dotés de visions, d'objectifs et de stratégies contradictoires. Ces luttes conditionnent non seulement l'élaboration des politiques publiques, mais également l'innovation dans les services. L'innovation dans les services se met habituellement en œuvre à une distance raisonnable des responsables de politiques et n'est pas systématiquement proposée dans le contexte de programmes politiques à l'approche descendante, mais elle dépend dans une certaine mesure de l'appui politique ou de l'acceptation des chefs politiques. Ainsi, pour émerger, les innovations en matière de services doivent faire l'objet d'autorisations et de légitimations politiques par rapport à l'ensemble des projets politiques. Or si ces projets politiques ne sont pas innovants, ils auront tendance à bloquer les innovations dans les services. Pourtant, les politiques novatrices ne sont pas seulement utiles à la promotion des innovations dans les services. Elles ont un objectif en soi. Une cible importante de l'innovation dans le secteur public est le développement de nouvelles visions, de nouveaux objectifs, de nouvelles stratégies politiques pour ce secteur. La pleine reconnaissance du rôle essentiel joué par les politiques novatrices et par l'innovation dans les services permet aux politiciens et aux autres intervenants impliqués dans l'élaboration des politiques d'exercer un rôle majeur dans l'amélioration de l'innovation dans le secteur public.

\section{LA CONCEPTUALISATION DE L'INNOVATION EN MATIÈRE DE POLITIQUES}

La manière de générer des idées créatives fait souvent l'objet de discussions visant à favoriser l'innovation, mais, comme il en est question dans la théorie de l'innovation, celle-ci nécessite bien plus que la simple expression de nouvelles idées. Il faut également mettre en œuvre et diffuser ces idées (Rogers, 1995; Mintrom et Vergari, 1998; Wejnert, 2002). Les nouvelles idées deviennent des innovations lorsqu'elles sont mises en œuvre et cette transformation d'une idée en une création utilisable et utilisée par plusieurs fait partie du processus créatif. La mise en œuvre exige le prototypage expérimental dans un processus de tâtonnements, alors que la diffusion demande l'adaptation vers d'autres contextes. De ce point de vue, l'innovation peut être définie comme la formulation, la mise en œuvre et la diffusion de nouvelles idées créatives (Sørensen et Torfing, 2011). La théorie de l'innovation signale également l'existence de différentes formes d'innovation, dont l'innovation dans 
les produits, dans les formes et procédures organisationnelles guidant certaines activités et dans la culture et les archétypes qui ajoutent de la valeur à certaines activités (Halvorsen et autres, 2005; Hartley, 2005). Comme l'illustre le tableau 1, ce phénomène fait également partie de l'innovation en matière de politiques.

\section{TABLEAU 1 : LES DIFFERENTS TYPES D'INNOVATION EN MATIERE DE POLITIQUES}

\begin{tabular}{l|l|l}
\hline PRODUITS & ORGANISATION ET PROCÉDURES & CULTURE \\
\hline $\begin{array}{l}\text { Politique avec nouveau } \\
\text { contenu et nouvelle forme } \\
\text { (problème, vision, objectif et } \\
\text { stratégie) }\end{array}$ & $\begin{array}{l}\text { Nouveaux cadres institutionnels } \\
\text { pour l'élaboration des politiques }\end{array}$ & $\begin{array}{l}\text { Nouvelle logique politique de ce } \\
\text { qui est approprié et nouvelle } \\
\text { perception des rôles }\end{array}$ \\
\hline
\end{tabular}

\section{L'INNOVATION DANS LES PRODUITS}

L'innovation en matière de produit transforme le contenu ou la forme d'une politique (Polsby, 1984). Les innovations sur le plan du contenu peuvent consister en la formulation, la mise en œuvre et la diffusion de nouvelles définitions d'un problème, de nouvelles visions, de nouveaux objectifs ou de nouvelles stratégies. Les politiques peuvent être qualifiées d'innovatrices lorsqu'elles décrivent de nouvelles perspectives politiques, redéfinissent des compréhensions bien établies d'un problème politique, fixent des objectifs inusités sur le plan des politiques ou proposent de nouvelles manières d'atteindre de tels objectifs. Ainsi, l'innovation en matière de contenu d'une politique a tendance à demander des remises en question discursives au sein desquelles des interprétations hégémoniques des politiques bien établies sont confrontées à des discours concurrentiels sur le plan des politiques (Howarth et Torfing, 2005). La position hégémonique des discours existants sur les politiques a tendance à résister au changement, et le succès des politiques novatrices dépend des remises en question occasionnées par les nouveaux besoins de la société et des capacités des acteurs politiques à exploiter ces besoins pour formuler, mettre en œuvre et diffuser des politiques novatrices (Kingdon, 1984). Une innovation de contenu d'une politique pourrait être un changement de perspective dans la prise en charge des personnes âgées, comme passer d'une perspective de soins vers la réhabilitation. Par ailleurs, l'innovation ne se limite pas aux modifications de contenu; elle peut concerner un changement de forme. Les politiques sont généralement produites sous la forme de longs documents écrits présentés en langage complexe et formel que bien peu de gens liront et comprendront. Cette façon d'exprimer, de condenser et de communiquer une vision, un objectif ou une stratégie risque de ne pas être une manière efficace de diffuser une nouvelle politique. L'innovation sur le plan de la forme d'une politique nécessite le choix d'un nouveau média pour diffuser le contenu auprès du public. Par exemple, une telle innovation pourrait être la diffusion du contenu d'une politique par le truchement d'interventions théâtrales, de textos ou de vidéos sur la plateforme YouTube.

\section{L'innovation sur le plan de l'organisation et des procédures}

Les politiques novatrices se trouvent également dans le développement de nouveaux contextes organisationnels pour l'élaboration, la formulation, la mise en œuvre et la diffusion des politiques. La vie politique au sein des démocraties libérales tend à être hautement 
organisée et réglementée par le truchement de règles détaillées et de procédures déterminant qui devrait décider quoi, où, quand et comment. Bien que ces procédures organisationnelles soient généralement conçues en référence au même modèle idéaltypique (par exemple le modèle du gouvernement représentatif), il existe de vastes variantes dans la manière dont elles sont mises en pratique d'un pays à l'autre (Lijphart, 1977 et 1999). L'innovation dans les organisations et les procédures politiques est importante, entre autres parce que les changements dans les cadres institutionnels de l'élaboration des politiques peuvent favoriser la capacité d'un système politique à innover sur le plan des produits. Les efforts en vue de changer des procédures ou des organisations politiques bien établies peuvent cependant être ralentis par la structure institutionnelle dépendante du chemin parcouru (Pierson, 2000) et parce que de tels changements ont tendance à intensifier la contestation politique en raison de leur effet sur la distribution du pouvoir entre intervenants, à l'avantage de certains et au détriment des autres (Roberts et Bradley, 1991). Les réformes de l'Union européenne constituent un exemple de ce type d'innovation lorsque de nouveaux traités négociés engendrent de nouvelles manières de préparer les rencontres en comités politiques au niveau national ou infranational et des changements dans la composition des activités de ces rencontres.

\section{L'innovation culturelle}

En terminant, l'innovation culturelle peut concerner le déploiement d'une nouvelle culture politique. Ce type d'innovation suppose l'institutionnalisation de nouvelles images décrivant la manière dont sont censés agir différents groupes d'intervenants au sein des processus politiques. On peut décrire une culture comme un ensemble de normes et de lignes directrices tacites définissant les façons correctes d'agir et d'interagir (Shein, 1992). Ces normes et lignes directrices renvoient à une logique plus ou moins cohérente de ce qui est approprié. Elles prennent la forme d'un système de signification fixe qui précise les différents rôles et positions des intervenants et qui spécifie comment ceux-ci peuvent se comporter de manière significative, normale et légitime dans certaines situations. Une culture politique offre un ensemble fixe de rôles et de positions par lesquels il est possible de pénétrer la vie politique. Chaque rôle (par exemple politicien, fonctionnaire, citoyen) reçoit des attributs précis qui lui permettent d'agir de certaines façons sur le plan politique (Weber, [1920] 1971; Almond et Verba, 1961). L'innovation dans une culture peut être précieuse, car elle ouvre vers la construction d'une nouvelle vision du monde, de nouvelles relations entre les intervenants et de nouvelles pratiques politiques. Il n'en demeure pas moins que les changements culturels sont complexes, car le sens et le rôle des modèles sont enchâssés dans des logiques fixes de ce qui est approprié, dans les perceptions de soi et dans les identités des intervenants, ainsi que dans les pratiques devenues des routines qui constituent la vie politique au jour le jour. Les innovations au sein d'une culture politique peuvent être initiées par des réformes du secteur public qui redistribuent les tâches entre les intervenants, amènent de nouveaux types d'intervenants au cœur du processus d'élaboration des politiques et installent de nouvelles procédures pour la formulation, la mise en œuvre et la diffusion des politiques. Exemple récent d'une innovation culturelle : la redéfinition des rôles des politiciens, des fonctionnaires et des citoyens qui a été faite dans le cadre du programme de réformes de la nouvelle gestion publique. Les politiciens sont devenus des directeurs de conseil d'administration, les fonctionnaires des gestionnaires stratégiques et les citoyens des consommateurs (Torfing et autres, 2012). Comme nous le verrons plus tard, les réformes de type nouvelle gouvernance publique déclenchent des innovations culturelles qui engendrent de nouvelles perceptions des rôles. 
Les tentatives d'innovation en matière de politiques peuvent viser l'innovation dans les produits, l'organisation ou la culture, mais des changements dans l'un de ces domaines ont tendance à déclencher des changements dans un autre. C'est à tout le moins le cas lorsque les innovations s'avèrent radicales. Une transformation radicale du contenu d'une politique peut exiger de nouveaux modes d'interaction entre les intervenants, et de tels changements peuvent forcer à réévaluer les perceptions des rôles des différents intervenants. De la même manière, les changements concernant la procédure ou l'organisation ou les changements dans la perception des rôles peuvent entraîner l'apparition de nouveaux contenus dans les politiques. Bref, nous définissons l'innovation politique comme la formulation, la mise en œuvre et la diffusion de nouvelles visions politiques, de nouveaux objectifs et stratégies en vue de la réalisation d'une société meilleure et comme les nouvelles formes, procédures et cultures politiques organisationnelles qui structurent le processus d'élaboration des politiques.

\section{LES POSSIBILITÉS DE LA COLLABORATION POUR DES POLITIQUES NOVATRICES}

Tout en gardant en tête les divers facteurs mentionnés dans la section précédente qui peuvent entraver les différents aspects de l'innovation dans les politiques, il est important de signaler que l'innovation en matière de politiques est un phénomène actif dans les systèmes politiques démocratiques. En s'inspirant de la récente théorie de l'innovation et de la théorie de la gouvernance, il est possible de déterminer trois importants moteurs des politiques novatrices: la hiérarchie, la concurrence et la collaboration. Les différentes approches des théories tendent à faire l'éloge de l'un de ces moteurs tout en négligeant les autres. Par opposition, nous considérons que les trois moteurs dépendent les uns des autres : la hiérarchie et la concurrence motivent les intervenants à innover alors que la collaboration engendre les innovations.

Les formes hiérarchiques de gestion, exercées par des chefs forts sur les plans politique et administratif, sont précieuses. Elles agissent comme des moteurs encourageant la création d'une politique novatrice. Les tentatives ascendantes de lancer des innovations politiques se produisent parfois, mais dans la plupart des cas de telles tentatives doivent, et c'est la règle, requérir l'autorisation des chefs politiques si elles veulent avoir la moindre chance de se voir couronnées de succès. Les pensées traditionnelles de la science politique et de la théorie de l'administration publique ont eu tendance à exagérer la capacité de transformation des formes hiérarchiques de gestion exercées par des chefs politiques (Weber, [1920] 1971; Schumpeter, 1946). La théorie des choix publics qui a inspiré la nouvelle gestion publique a sous-estimé le rôle que peut jouer la règle hiérarchique dans la promotion de l'innovation dans le secteur public (Downs, 1967; Niskanen, 1975) et il a été ainsi également de la théorie de la mise en œuvre (Pressman et Wildavski, 1973). Bien que les chefs politiques soient limités, entre autres facteurs par leur manque de connaissances, ils ont souvent les compétences et la position qui leur permettent de répandre des récits sur des programmes et de possibles nouveaux avenirs; les leaders politiques ont un accès privilégié aux médias de masse, et la rhétorique et la persuasion sont au cœur de leur profession (Willner, 1985; Tucker, 1995). De plus, les chefs politiques détiennent les pouvoirs formels d'exercer une pression politique, légale et financière ou d'ordonner à des intervenants pertinents de s'engager dans une innovation politique. De cette manière, ils jouent un rôle important en tant que catalyseurs de l'innovation politique (Ansell et Gash, 2012).

La concurrence est un autre important vecteur de l'innovation politique. L'une des pierres angulaires des théories de la démocratie représentative est l'existence d'une lutte 
pour le pouvoir entre les chefs politiques. Dans le cadre de cette concurrence, les politiciens subissent une pression constante les poussant à établir de nouveaux programmes politiques innovateurs qui convaincront les citoyens de voter pour eux (Polsby, 1984). La même école de pensée a engendré le paradigme de la nouvelle gestion publique en vue d'améliorer l'efficacité et le rendement de l'innovation dans les services publics par le truchement d'une concurrence entre les fournisseurs de ces services (Osborne et Gaebler, 1993). Alors que les théories de la démocratie représentative et le paradigme de la nouvelle gestion publique sont exagérément optimistes (Rowley, 1987) dans leurs attentes face à la capacité innovante des formes concurrentielles de gestion, le paradigme de la nouvelle gouvernance publique a tendance à se montrer trop pessimiste (Hartley, 2005; Osborne, 2010). Lorsque d'habiles chefs politiques racontent une histoire, promulguent des lois ou donnent des ordres qui ne parviennent pas à stimuler le degré désiré d'innovation en matière de politiques, la concurrence peut ajouter une pression additionnelle qui agit sur les intervenants pour les pousser à s'engager dans l'innovation - qu'ils soient des employés du secteur public, des agences, des entreprises privées, des organismes bénévoles ou de simples citoyens. Comme la hiérarchie, la concurrence est un élément clé de l'innovation politique; l'innovation étant une affaire pénible et risquée, il est très tentant pour les intervenants individuels comme pour les groupes de s'en tenir aux habitudes quotidiennes. La hiérarchie et la concurrence peuvent placer les intervenants dans des situations où l'innovation représente l'option la plus favorable, malgré les coûts et les risques encourus.

Bien que la hiérarchie et la concurrence soient importantes pour motiver les intervenants à s'engager dans l'innovation, ces deux formes de gestion ne contribuent pas nécessairement au développement de politiques novatrices. Elles peuvent même, d'une certaine manière, bloquer l'innovation. Les hiérarchies rigides sont susceptibles d'empêcher la communication verticale entre les chefs politiques, les employés du secteur public et les clientèles. Il en va de même pour la concurrence féroce opposant des partis politiques ou des partis et d'autres intervenants visant à influencer le processus de prise de décision. Cette contrainte sur les communications verticales et horizontales réduit la capacité d'innovation sur le plan des politiques de la même manière qu'elle étouffe le dialogue politique et la contestation, le partage des connaissances et des idées, ainsi que les actions partagées entre les intervenants concernés. Cet effet négatif, que peuvent avoir le règne hiérarchique et la concurrence sur la capacité à innover, a été remarqué par les récentes analyses de la théorie de l'innovation et de la théorie de la gestion, qui mettent l'accent sur le rôle important que peut jouer la collaboration dans la formulation, la mise en œuvre et la diffusion des innovations (Teece, 1992; Edquist et Hommen, 1999; Borins, 2001; Gloor, 2005; Nambisan, 2008; Bommert, 2010). Ces théories remettent en question l'hypothèse selon laquelle les innovations sont produites par des héros isolés, tels les entrepreneurs créatifs, les chercheurs inventifs ou les chefs courageux. Les nouvelles théories prétendent montrer que dans la vraie vie les innovations sont la plupart du temps le résultat de collaborations entre un grand nombre d'intervenants dotés de compétences, d'expériences, de visions et d'hypothèses complémentaires dans un domaine donné. La collaboration est fondamentale parce qu'un intervenant isolé a rarement la capacité de générer une idée nouvelle, de la mettre en œuvre et de la diffuser. Par ailleurs, la collaboration peut fonctionner comme un instrument susceptible d'engendrer des dérangements et de déstabiliser une scène politique fixée; elle peut ainsi conduire à des tentatives de gouvernance dans de nouvelles avenues.

La collaboration peut servir de moteur à l'innovation dans toutes ses phases de développement (Freeman, 1991; Sørensen et Torfing, 2011) et réunit une grande variété d'intervenants dans un domaine politique donné. La collaboration : 
- encourage l'agrégation d'une vaste gamme d'idées d'intervenants avec des perspectives variées et stimule la création de nouvelles idées par un processus de destruction créative et de réarticulation d'une politique;

- augmente la capacité de mettre en œuvre une politique en fournissant aux responsables de la mise en œuvre de l'idée novatrice une compréhension complète de celleci et un profond sens de l'appartenance et de l'engagement envers elle;

- favorise la diffusion d'une innovation politique par l'entremise d'ambassadeurs de l'innovation qui souhaitent déployer des efforts pour communiquer les bienfaits d'une innovation politique couronnée de succès à laquelle ils ont participé à l'élaboration. Cette innovation pourrait être adaptée par d'autres intervenants politiques.

Pour résumer, les trois formes de gouvernance peuvent contribuer à promouvoir l'innovation politique de façon complémentaire : la hiérarchie peut amorcer la recherche de nouvelles politiques; la concurrence peut motiver les intervenants à s'engager dans les tentatives d'innovation; la collaboration peut favoriser l'invention d'innovations. La reconnaissance du rôle crucial de la collaboration dans les processus d'innovation signifie que le règne hiérarchique et la gouvernance concurrentielle doivent être exercés de manière à favoriser la collaboration entre ceux qui ont la capacité d'innover dans une politique donnée. Le règne hiérarchique doit enclencher la collaboration par la création de lieux où peuvent se réunir des intervenants dotés des ressources et des mandats nécessaires leur permettant de s'engager dans la formulation, la mise en œuvre ou la diffusion de nouvelles innovations politiques. La concurrence, quant à elle, doit être conçue de manière à motiver certains acteurs à collaborer, car la concurrence produit des interdépendances.

Pour cette raison, l'augmentation des innovations sur le plan des politiques exige la présence d'un leader politique habile qui prendrait en charge la tâche de promouvoir l'innovation politique par la conception stratégique de divers espaces de collaboration pour des politiques commandées par des pressions hiérarchiques et des forces concurrentielles. Cette tâche regroupe ceux qui cherchent à stimuler l'innovation politique - qu'ils soient des politiciens, des cadres de direction ou d'autres intervenants dans une position leur permettant de concevoir des espaces de collaboration innovante - dans trois catégories de rôles : le convocateur, le facilitateur et le catalyseur (Ansell et Gash, 2012; O’Toole, 1997). Le convocateur mandate et motive les intervenants concernés afin que ceux-ci participent au processus de collaboration innovante par la formation d'interdépendances; le facilitateur assiste l'interaction de diverses façons pour stimuler la collaboration; le catalyseur crée des dérangements qui augmentent la volonté et la capacité des collaborateurs à innover. Bien que ces trois rôles de leadership puissent être joués en partie par d'autres que les politiciens (par exemple par des administrateurs publics ou d'importants intervenants), les politiciens, qui auront éventuellement à valider les innovations, doivent jouer un rôle clé dans la direction de ces processus de collaboration innovante.

\section{LES OBSTACLES À LA COLLABORATION INNOVANTE}

Comme il en a été question ci-dessus, les contextes institutionnels traditionnels et les rôles et pratiques de routine peuvent constituer des obstacles à la croissance des différents aspects de l'innovation en matière de politiques. Il en va ainsi tant pour les innovations de forme et de contenu des politiques (produit politique) et le cadre institutionnel par lequel les politiques sont formulées, mises en œuvre et diffusées (organisation et procédures), que pour la culture politique (perception des rôles et logique de ce qui est approprié). Il existe 
de nombreuses barrières potentielles à la collaboration innovante dans les démocraties occidentales contemporaines, car le contexte institutionnel, les pratiques et les perceptions des rôles sont inspirés par des modèles de gestion qui célèbrent la séparation des pouvoirs et la division des tâches plutôt que la collaboration et tendent à isoler l'élaboration des politiques à une seule étape ou à un bref instant dans la chaîne de décision rationnelle (Sørensen, 2007; Torfing et autres, 2012). Les possibles blocages auxquels fait face la collaboration innovante en matière de politiques sont évoqués dans le tableau 2.

\section{TABLEAU 2: LES OBSTACLES POTENTIELS A LA COLLABORATION INNOVANTE EN MATIERE DE POLITIQUES}

\begin{tabular}{l|l|l|}
$\begin{array}{l}\text { OBSTACLES AUX PRODUITS } \\
\text { POLITIQUES }\end{array}$ & $\begin{array}{l}\text { OBSTACLES SUR LE PLAN DE } \\
\text { L'ORGANISATION OU DES } \\
\text { PROCÉDURES }\end{array}$ & $\begin{array}{l}\text { OBSTACLES SUR LE PLAN DE LA } \\
\text { CULTURE POLITIQUE }\end{array}$ \\
\hline $\begin{array}{l}\text { Le contenu d'une politique est } \\
\text { fréquemment construit de } \\
\text { réactions aux crises actuelles } \\
\text { plutôt que de réponses } \\
\text { proactives aux défis de } \\
\text { demain. }\end{array}$ & $\begin{array}{l}\text { L'élaboration des politiques est } \\
\text { principalement organisée sous } \\
\text { forme d'activité interne et } \\
\text { n'implique que les politiciens et } \\
\text { l'administration. }\end{array}$ & $\begin{array}{l}\text { Les images traditionnelles } \\
\text { représentant la manière } \\
\text { d'élaborer les politiques } \\
\text { découragent les interactions } \\
\text { avec des intervenants de } \\
\text { l'extérieur. }\end{array}$ \\
$\begin{array}{l}\text { Les politiques sont souvent } \\
\text { présentées sous une forme } \\
\text { qui ne correspond pas à ceux } \\
\text { qui seront chargés de les } \\
\text { mettre en œuvre et de les } \\
\text { diffuser. }\end{array}$ & $\begin{array}{l}\text { Si des facteurs extérieurs sont } \\
\text { impliqués, ils ont tendance à } \\
\text { l'être à un stade très tardif du } \\
\text { processus d'élaboration des } \\
\text { politiques. }\end{array}$ & $\begin{array}{l}\text { Les perceptions fixées des rôles } \\
\text { définissent l'élaboration des } \\
\text { politiques comme une tâche } \\
\text { dévolue aux seuls politiciens. }\end{array}$ \\
\hline
\end{tabular}

Les politiques élaborées en guise de réponses aux crises quotidiennes, plutôt que sous la forme de tentatives proactives de prendre en charge des questions à long terme ou de développer de nouveaux horizons, objectifs ou stratégies de politiques tournés vers l'avenir, risquent d'engendrer de faibles améliorations technocratiques au lieu de révolutions radicales. De plus, la tendance à associer des intervenants pertinents sera faible, ce qui peut entraver les efforts de mise en œuvre et de diffusion des innovations politiques.

Lorsque l'élaboration des politiques est principalement organisée en tant qu'activité interne au sein des gouvernements, des Parlements, des comités politiques ou des hôtels de ville, le niveau de dialogue, de partage des connaissances et de la collaboration entre politiciens et intervenants dotés de ressources et d'expériences pertinentes sera limité et la capacité du système politique d'innover sur le plan des politiques en sera réduite. La plupart des systèmes politiques possèdent des mécanismes qui permettent aux intervenants externes de commenter les nouveaux programmes de réformes politiques, mais les réactions ne parviennent en général que tardivement aux politiciens dans le processus, par le truchement d'audiences par exemple, avec pour effet qu'il est peu envisageable de procéder à des changements.

La majorité des modèles contemporains de démocratie représentative demandent aux autorités publiques en général et aux politiciens en particulier qu'ils maintiennent une distance sécuritaire entre eux et les intervenants sociaux afin d'éviter d'être victimes de lobbyisme ou d'être emprisonnés dans des luttes de pouvoir qui pourraient les distraire de leur 
rôle de serviteurs de l'intérêt général de la société. L'incapacité d'innover sur le plan des politiques est encore augmentée par la vision largement consensuelle selon laquelle l'élaboration des politiques est une tâche solitaire destinée aux politiciens et réduisant par le fait même les autres intervenants (tels les bureaucrates de faible niveau, les citoyens, les entreprises privées et les organisations de bénévoles) dans un rôle passif de destinataires des innovations politiques. Les théories descriptives des gouvernements représentatifs reconnaissent que d'autres intervenants remplissent un rôle dans l'élaboration des politiques, mais voient cet état de fait comme une déviance regrettable par rapport à la situation idéale (Sartori, 1987). Ainsi, bien que l'innovation en matière de politiques associe parfois des intervenants autres que les politiciens, le phénomène se produit malgré ce qui est prêché par le système hégémonique de perception des rôles et de logique de ce qui est approprié.

\section{DES EXPÉRIMENTATIONS AVEC LA COLLABORATION INNOVANTE EN MATIÈRE DE POLITIQUES : UN NOUVEAU RÔLE POUR LES POLITICIENS}

Il existe donc de nombreux obstacles à la collaboration innovante en matière de politiques dans le secteur public en raison de la manière dont le processus d'élaboration des politiques est majoritairement perçu et institutionnalisé au sein des démocraties représentatives. Les efforts pour éliminer les entraves à l'innovation visent alors le développement (1) de nouvelles compréhensions de ce qu'est une politique et de la manière de la représenter; (2) de nouvelles procédures et de nouveaux cadres organisationnels pour l'élaboration des politiques permettant de réunir les autorités publiques et les intervenants concernés dans une tentative de formulation, de mise en œuvre et de diffusion des politiques; (3) de nouveaux archétypes pour les politiciens, les administrateurs publics, les citoyens, les entreprises privées et les organisations de bénévoles qui guideraient leur participation dans la collaboration innovante en matière de politiques.

L'un des plus importants obstacles à l'innovation politique a trait au rôle assigné aux politiciens. Bien que d'autres facteurs s'avèrent essentiels dans la collaboration innovante en matière de politiques, les politiciens ont un rôle particulièrement important à jouer, car les institutions de la démocratie représentative les autorisent à être de légitimes créateurs de politiques. Quel rôle pourraient-ils assumer dans un processus de collaboration innovante? Comme il en a été question plus haut, leur poste traditionnel de politiciens souverains offre peu de prise sur ce que pourrait devenir ce rôle. Or le besoin de conception d'espaces favorisant la collaboration innovante peut nous indiquer une avenue. Bien que les politiciens puissent ne pas être en position de prendre seuls en charge les rôles de convocateurs, de facilitateurs ou de catalyseurs de la collaboration innovante, leur posture en tant qu'autorité politique légitime suggère qu'ils pourraient définitivement prendre une part active en tant que meneurs des processus d'innovation des politiques. Dans l'exercice de ce rôle, ils peuvent clairement bénéficier de l'appui d'administrateurs publics compétents sur le plan du leadership ainsi que d'intervenants importants jouissant de capacités et de ressources pertinentes. Le leadership, dans un tel contexte, représente une entreprise collaborative. Cependant, les politiciens devraient également exercer le rôle de participants dans le processus de collaboration innovante en matière de politiques, car eux-mêmes sont des créateurs de politiques désignés par les institutions. De cette manière, ils peuvent tester et développer leurs visions et leurs idées à propos d'une question de politique dans le cadre d'un dialogue ou d'une remise en question avec des intervenants issus de différents milieux, tels des experts, des bureaucrates d'échelon inférieur, des citoyens et des intervenants du secteur privé (Sørensen, 2006; Torfing et autres, 2012). 
Les résultats de deux études de cas d'expériences de collaboration pour des politiques innovantes au Danemark sont intéressants dans ce contexte, car les deux études avaient pour objectif de créer de nouveaux produits politiques par la modification du contexte organisationnel et des procédures d'élaboration des politiques. Dans ces expériences, les politiciens étaient appelés à jouer de nouveaux rôles. Les deux études de cas sont également intéressantes, car elles suggèrent que le degré de mise en œuvre et de diffusion d'une politique dépend du degré d'implication des politiciens comme leaders et participants. De plus, elles révèlent les relations existantes entre l'innovation sur le plan du produit, les changements dans les procédures et le contexte organisationnels de l'élaboration des politiques et la perception des rôles qu'ont les différents acteurs, c'est-à-dire les politiciens. Le tableau 3 résume les rôles assignés aux politiciens dans les deux projets.

\section{TABLEAU 3 : LES RÔLES ASSIGNÉS AUX POLITICIENS DANS UN CONTEXTE DE COLLABORATION INNOVANTE}

\begin{tabular}{|l|c|c|c|c|}
\hline & CONVOCATEUR & FACILITATEUR & CATALYSEUR & PARTICIPANT \\
\hline Municipalité d'Albertslund & + & $(+)$ & $(-)$ & + \\
\hline Conseil de l'éthique & - & - & - & $(+)$ \\
\hline
\end{tabular}

\section{Une collaboration innovante à la municipalité d'Albertslund}

En 2010, la municipalité d'Albertslund a mis en œuvre un projet visant à élaborer une politique innovante d'implication des citoyens par l'aménagement d'un espace de collaboration en matière de politiques. Un collègue et moi avons observé le processus du début à la fin, y compris les processus de mise en œuvre et de diffusion. Les données ont été recueillies par le truchement d'observations de réunions et autres événements, par l'étude de documents et à l'aide d'entrevues de recherche conduites auprès des intervenants impliqués ${ }^{1}$. Le projet a été mis de l'avant par le conseil municipal qui a décidé de rompre avec la traditionnelle élaboration de politiques dans les comités habituels et a formé à la place un comité $a d$ hoc composé de six politiciens, six citoyens et trois fonctionnaires. Le comité avait un an pour préparer une proposition de politique et devait tenir quatre réunions. Le comité a cependant choisi de travailler autrement et d'une manière plus expérimentale. Il a ainsi été décidé d'inviter différents experts en politique et de tenir un "camp de l'innovation » et un atelier associant les parties prenantes concernées en plus d'organiser un certain nombre de réunions plus traditionnelles, bien que ces dernières soient graduellement passées de réunions formelles avec ordres du jour, modérateurs et procès-verbaux à des ateliers avec travaux de groupes et cartographie conceptuelle. Résultat de ce processus : les relations et les perceptions des rôles des participants - citoyens, politiciens et administrateurs - ont radicalement changé. Les citoyens ont vu la municipalité d'une nouvelle manière et ont commencé à se considérer comme des coproducteurs compétents d'idées en matière de politiques, alors que les politiciens ont découvert qu'il était possible de discuter des questions directement avec les citoyens et les autres intervenants et que cela leur donnait accès à cer-

1 L'étude fait l'objet d'un reportage détaillé dans Agger et Sørensen (à paraître). 
tains points de vue qu'il leur était possible de rapporter par la suite au conseil municipal. Les administrateurs ont également redéfini leur position; ils ont commencé à se voir comme des participants au processus d'élaboration des politiques et non plus comme des responsables du contrôle des activités. Le conseil municipal a participé au camp sur l'innovation qui a eu lieu à mi-chemin dans l'année. À la fin du projet, le comité ad hoc a présenté une proposition de politique à deux assemblées du conseil municipal et la proposition a été adoptée sans modifications. Le produit était un document politique qui reformulait la définition du problème et l'objectif décrits par la commission originale. Bien que la forme de ce document politique n'ait pas été radicalement différente des autres politiques, il était augmenté d'une certaine quantité de vidéos mettant en scène des entrevues de citoyens au sujet de l'implication citoyenne et d'un manuel décrivant la manière dont les employés peuvent impliquer les citoyens dans les processus de décision.

Lequel des postes mentionnés plus haut les politiciens ont-ils donc adopté dans le processus de collaboration innovante? Ils ont d'abord joué un rôle clé en tant que convocateurs du processus de collaboration innovante. Ils ont amorcé le processus en définissant l'ordre du jour général par la formulation de la commission et en décidant de la composition du comité, ainsi que des échéanciers et de la forme du produit politique. Le rôle de facilitateur a cependant été délégué aux trois administrateurs, bien que ceux-ci aient tenté d'impliquer différents politiciens et citoyens dans la préparation et l'organisation des réunions et autres activités. Le rôle de catalyseur a été principalement réservé aux administrateurs qui ont planifié les différents ateliers, camps et autres interventions en collaboration avec les parties prenantes et consultants externes. Le maire, qui était membre du comité, a participé d'une certaine manière en autorisant les événements catalyseurs. En tant que leaders politiques, les politiciens semblent avoir joué un rôle modeste dans le processus, mais ils ont été très actifs comme participants au processus. C'était en particulier le cas des six membres du comité, mais également des autres politiciens du conseil municipal impliqués dans le processus du début à la fin.

\section{Une collaboration innovante au sein du Conseil de l'éthique}

En 2011, un groupe de réflexion, le «Conseil de l'éthique », a lancé un processus de collaboration innovante afin de développer un document de politique générale sur l'usage de la force dans les traitements psychiatriques. Un collègue et moi avons observé le processus d'élaboration de la politique qui a duré un an. Comme dans l'étude d'Albertslund, les données incluent des observations des réunions et d'autres événements, l'étude de documents et des entrevues de recherche en compagnie des acteurs concernés ${ }^{2}$. Le Conseil de l'éthique a été formé par le Parlement national danois en 1987 avec pour mission de conseiller le Parlement sur des points de politiques touchant des questions d'éthique liées à la science. Le Conseil est dirigé par un conseil d'administration nommé par le Parlement et différents ministères. Le Conseil a instauré le projet de collaboration innovante afin d'adopter un rôle plus proactif pour mieux se préparer à offrir des avis et pour améliorer sa capacité à retenir l'attention des politiciens. L'idée de base était de réunir tous les intervenants pertinents pour débattre de la question de l'usage de la force dans le traitement des patients psychiatriques et de ce qui pourrait être fait pour en réduire l'incidence. L'expérience a eu pour résultat une innovation dans le produit politique, dans les formes d'organisation et de pro-

2 Pour de plus amples détails, voir Sørensen et Waldorff (à paraître). 
cédures et dans la culture et la perception des rôles des intervenants impliqués. Toutefois, comme nous le verrons, la portée du projet sur l'élaboration des politiques est demeurée marginale, car les politiciens n'étaient pas suffisamment impliqués dans le processus.

Le produit politique s'avérait innovateur puisque le Conseil de l'éthique avait radicalement changé sa compréhension de ce qui entraîne l'usage de la force dans le contexte des traitements psychiatriques, passant d'une question de restrictions économiques et d'un manque d'encadrement légal à une situation causée par la culture organisationnelle et professionnelle présente dans les instituts psychiatriques. Ce changement dans la définition du problème a déclenché une nouvelle compréhension du groupe cible. À partir d'une posture axée plus ou moins exclusivement sur la communication d'avis aux politiciens sous forme de rapports politiques, un effort a été consenti envers la production de dossiers et de documents vidéo visant les personnes travaillant dans les établissements psychiatriques. Des expériences innovantes ont également été menées dans la manière dont les projets ont été organisés par le Conseil de l'éthique. En temps normal, le Conseil soumet des propositions de politiques en discutant à l'interne avec les experts du conseil d'administration sur la base de propositions reçues par un secrétariat. À cette occasion, le Conseil a tenté quelque chose de nouveau. Une firme de consultants privée a été engagée pour prendre les commandes dans l'organisation du projet sous forme de processus de collaboration innovante. Il a été décidé de tenir trois ateliersthéâtre, ainsi qu'une conférence de clôture présentée à Christianborg, où se trouve le Parlement danois. Les quatre événements ont été planifiés comme une série de rencontres entre l'entreprise et certains membres du Conseil de l'éthique. Un nombre croissant d'intervenants ont participé à ces ateliers, passant de 15 à 50, et 200 participants ont assisté à la conférence finale. La plupart étaient des professionnels venus des instituts psychiatriques, mais des politiciens, d'anciens patients, ainsi que quelques organisations de bénévoles ont également été invités. Bien qu'il soit difficile d'évaluer à quel point les quatre événements ont influé sur la relation entre les intervenants ainsi que sur leurs perceptions de leurs rôles, le processus a certainement eu une influence sur la culture au sein du Conseil de l'éthique. À l'origine cantonné dans une approche d'experts scientifiques sur les questions d'éthique, le Conseil a commencé à comprendre la valeur et la pertinence des expériences des intervenants dans la considération des questions d'éthique.

Le projet de collaboration a certes innové en ce qui a trait au produit politique, aux formes et procédures organisationnelles employées par le Conseil de l'éthique, à sa culture et à sa perception de lui-même, mais il n'est pas parvenu à accomplir son plein potentiel en raison du rôle marginal tenu par les politiciens. En fait, les politiciens n'ont pas assumé le rôle de meneurs politiques; ils n'ont ni lancé les initiatives, ni facilité le projet, ni servi de catalyseurs. Ils se sont contentés de participer à certains des événements. Cette façon d'agir signifiait que la politique ne serait ni mise en œuvre, ni répandue au sein du système politique plus vaste. Le manque de leadership politique a fait en sorte que les politiciens ne se sentaient pas concernés par le processus ou par ses résultats. Ils n'étaient guère plus que des visiteurs dans le processus d'innovation, sans engagement face à la tâche de le présenter au système politique. Ce manque de leadership est apparu clairement à la réunion à laquelle les documents de politique devaient être présentés aux divers comités parlementaires. Aucun politicien ne s'est présenté et la réunion a été annulée. 


\section{CONCLUSION}

Dans le cadre des efforts actuels déployés en vue de favoriser l'innovation dans le secteur public, un intérêt limité a été porté à la question de l'innovation en matière de politiques publiques. Cet article a tenté d'insérer ce type d'innovation à l'ordre du jour de la recherche et a montré que malgré l'importance de la hiérarchie et de la concurrence comme moteurs de l'innovation politique, la collaboration est capitale pour développer de nouveaux produits politiques, de nouveaux contextes et procédures organisationnels pour l'élaboration des politiques et pour l'innovation dans notre culture politique. Il existe d'importantes entraves à la collaboration innovante sur le plan des politiques, tels les discours hégémoniques, les choix de politiques à structures dépendantes, les pratiques routinières et la perception des rôles (l'image des politiciens en tant que décideurs souverains les empêche de prendre en charge le rôle de leaders politiques et de participants dans les collaborations innovantes en matière de politiques). Les études de cas de deux expériences danoises de collaboration innovante montrent que le degré d'implication des politiciens comme leaders politiques et participants dans l'innovation politique est décisif, il détermine le succès ou l'échec de la collaboration. Bien que l'innovation en matière de politiques puisse se développer en l'absence des politiciens ou avec des politiciens impliqués en tant que participants plus ou moins marginaux, la mise en œuvre et la diffusion des politiques exigent des politiciens qu'ils remplissent le rôle de leaders. De plus, les études de cas montrent les liens unissant l'innovation dans les produits, l'innovation dans les procédures et l'organisation et l'innovation culturelle. L'innovation dans l'un de ces domaines semble avoir un effet d'entraînement sur les autres.

\section{BIBLIOGRAPHIE}

Agger, A. et E. Sørensen (à paraître). « Enhancing Collaborative Policy Innovation: A Case Study from a Danish Municipality», dans C. Ansell et J. Torfing (dir.), Public Innovation and Collaboration Through Design, Londres, Routledge.

Almond, G. et S. Verba (1961). The Civic Culture: Political Attitudes and Democracy in Five Nations, Washington D.C., Georgetown University Press.

Ansell, C. et A. Gash (2012). "Stewards, Mediators, and Catalysts: Towards a Model of Collaborative Leadership », The Innovation Journal, vol. 17, n 1, art. 7.

Bland, T. et autres (2010). "Enhancing Public Sector Innovation: Examining the NetworkInnovation Relationship », Public Sector Innovation Journal, vol. 15, no 3, p. 1-25.

Bommert, B. (2010). "Collaborative Innovation in the Public Sector», International Public Management Review, vol. 11, nº1, p. 15-33.

Borins, S. (2001). « Encouraging Innovation in the Public Sector », Journal of Intellectual Capital, vol. 2, n 3, p. 310-319.

Considine, M., J. Lewis et D. Alexander (2009). Networks, Innovation and Public Policy, Basingstoke, Palgrave Macmillan.

Damanpour, F. (1991). «Organizational Innovation: A Meta-analysis of Effects of Determinants and Moderators », Academy of Management Journal, vol. 34, n 3, p. 555-590.

Dente, B., L. Bobbio et A. Spada (2005). «Government or Governance of Urban Innovation? », DISP The Planning Review, vol. 41, nº 162, p. 41-52. 
Downs, A. (1967). Inside bureaucracy, Boston, Little, Brown.

Edquist, C. et L. Hommen (1999). « Systems of Innovation: Theory and Policy for the Demand Side », Technology in Society, vol. 21, n 1, p. 63-79.

Eggers, B. et S. Singh (2009). The Public Innovators Playbook, Washington D.C., Harvard Kennedy School of Government.

Freeman, C. (1991). « Networks of Innovators: A Synthesis of Research Issues », Research Policy, vol. 20, no 5, p. 499-514.

Gloor, P. A. (2005). Swarm Creativity: Competitive Advantage through Collaborative Innovation Networks, Oxford, Oxford University Press.

Halvorsen, T. et autres (2005). «On the Differences Between Public and Private Sector Innovation », Publin Report, no D9.

Hartley, J. (2005). "Innovation in Governance and Public Service: Past and Present», Public Money \& Management, vol. 25, nº 1, p. 27-34.

Hood, C. (1991). «A Public Administration for all Seasons? », Public Administration, vol. 69, no 1, p. 1-19.

Howarth, D. et J. Torfing (2005) (dir.). Discourse Theory in European Politics, Londres, Palgrave.

John, P. et G. Stoker (2008). «Design Experiments: Engaging Policy Makers in the Search for Evidence About What Works », Political Studies, vol. 57, n² 2, p. 356-373.

Kelman, S. (2005). Unleashing Change: A Study of Organizational Renewal in Government, Washington D.C., Brookings Institution.

Kingdon, J. W. (1984). Agendas, Alternatives, and Public Policies, Boston, Little, Brown.

Lijphart, A. (1999). Patterns of Democracy, New Haven, Yale University Press.

Lijphart, A. (1977). Democracy in Plural Societies: A Comparative Exploration, New Haven, Yale University Press.

Lundvall, B. Å. (1985). Product Innovation and User-producer Interaction, Aalborg, Aalborg University Press.

Mintrom, M. et S. Vergari (1998). "Policy Networks and Innovation Diffusion », Journal of Politics, vol. 60, no 1, p. 126-148.

Mulgan, G. et D. Albury (2003). «Innovation in the Public Sector», Working Paper, Strategy Unit, UK Cabinet Office, www.sba.oakland.edu/faculty/mathieson/mis524/resources/readings/ innovation/innovation in the public sector.pdf (page consultée le 20 août 2013).

Nambisan, S. (2008). Transforming Government through Collaborative Innovation, Washington D.C., Harvard Kennedy School of Government.

Newman, J., J. Raine et C. Skelcher (2001). « Transforming Local Government: Innovation and Modernization », Public Money \& Management, vol. 21, n² 2, p. 61-68.

Niskanen, W. A. (1987). "Bureaucracy », dans C. K. Rowley (dir.), Democracy and Public Choice, Oxford, Basil Blackwell, p. 135-140.

Niskanen, W. A. (1975). "Bureaucrats and Politicians », Journal of Law and Economics, vol. 18, $\mathrm{n}^{\circ} 3$, p. 617-43.

Osborne, S. (2010). The New Public Governance?, New York, Routledge. 
Osborne, D. et T. Gaebler (1993). Reinventing Government: How the Entrepreneurial Spirit is Transforming the Public Sector, Reading, Addison-Wesley.

O’Toole, L. J. (1997). «Implementing Public Innovations in Network Settings », Administration and Society, vol. 29, no 2, p. 115-138.

Pierson, P. (2000). «Increasing Returns, Path Dependence, and the Study of Politics », American Political Science Review, vol. 94, nº 2, p. 251-267.

Polsby, N. W. (1984). Political Innovation in America: The Politics of Policy Initiation, New Haven, Yale University Press.

Pressman, J. et A. Wildavsky (1973). Implementation, San Francisco, University of California Press.

Roberts, N. C. et R. T. Bradley (1991). "Stakeholder Collaboration and Innovation », Journal of Applied Behavioral Science, vol. 27, n 2, p. 209-227.

Rogers, E. M. (1995). Diffusion of Innovation, New York, Free Press.

Rowley, C. K. (dir.) (1987). Democracy and Public Choice, Oxford, Basil Blackwell.

Sartori, G. (1987). The Theory of Democracy Revisited, Chatham, Chatham House Publishers.

Schumpeter, J. (1946). Economic Theory and Entrepreneurial History, Cambridge, Harvard University Press.

Shein, E. (1992). Organizational Culture and Leadership: A Dynamic View, San Francisco, Jossey-Bass.

Sørensen, E. (2012). "Governance and innovation in the public sector», dans David Levi-Faur (dir.) ,The Oxford Handbook of Governance, New York, Oxford University Press, p. 215-227.

Sørensen, E. (2007). «Democratic Theory as a Frame for Decision Making: The Challenges by Discourse Theory and Governance Theory », dans G. Morcol (dir.), Handbook of Decision Making, New York, Marcel Dekker, p. 151-167.

Sørensen, E. (2006). « Metagovernance the Changing Role of Politicians in Processes of Democratic Governance », The American Review of Public Administration, vol. 36, no 1, p. 98-114.

Sørensen, E. et S. B. Waldorff (à paraître). 'Enhancing Politicians' Capacity for Political Leadership through Collaborative Policy Innovation, Paper presented at the IRSPM-conference in Prague, avril 2013.

Sørensen, E. et J. Torfing (2011). «Enhancing Innovation in the Public Sector », Administration and Society, vol. 43, no 8, p. 842-868.

Teece, D. J. (1992). « Competition, Cooperation, and Innovation », Journal of Economic Behavior \& Organization, vol. 18, no 1, p. 1-25.

Tucker, R.C. (1995). Politics as leadership, Columbia, University of Missouri.

Torfing, J. et autres (2012). Interactive Governance: Advancing the Paradigm, Oxford, Oxford University Press.

Warren, M. E. (2009). "Governance-Driven Democratization », Critical Policy Analysis, vol. 3, no 1, p. 3-13.

Weber, M. ([1920] 1971). Makt og Byråkrati, Oslo, Gyldendal Norsk Forlag.

Wejnert, B. (2002). «Integrating Models of Diffusion of Innovations: A Conceptual Framework », Annual Review of Sociology, vol. 28, p. 297-326.

Willner, A. R. (1985). The Spellbinders. Charismatic Political Leadership, Yale, Yale University Press. 